\title{
Comparison of Lower Limb Muscle Activation by Various Forms of Color Information during Gait
}

\author{
Sang-Yeol Lee, PT, PhD ${ }^{1)}$, Su-Kyoung Lee, PT, MS'2), Kyung-Jin Ha, PT, MS ${ }^{3)}$, \\ Jae-Hoon $\mathrm{Kim}^{2)}$, Yeon-Ju Kim ${ }^{2)}$, JeOng-Yun Kim${ }^{2)}$, KyeOng-Mi SeO ${ }^{2)}$, \\ Jin-SEONG PARK, PT), SEON-Chil, Kim, PhD ${ }^{5}$ ) \\ 1) Department of Physical Therapy, College of Science, Kyungsung University \\ 2) Department of Physical Therapy, Gimhae College: Sambang-dong, Gimhae, Gyeongsangnam-do, San \\ 77-9, Republic of Korea.TEL: +82 55-320-1735, FAX: +82 54-442-8953,E-mail:holeintwo@nate.com \\ 3) Department of Physical Medicine and Rehabilitation, Dong-a University Medical Center \\ 4) Rehabilitation Center, Seoho Hospital \\ 5) Department of Radiologic Technology, Daegu Health College
}

\begin{abstract}
Purpose] The purpose of this study was to evaluate the influence of lower limb muscle activation according to various forms of color information during walking. [Methods] This study was performed on 20 healthy adults. Lower limb muscles were measured with an MP 36 system(Biopac System Inc., Goleta, CA, USA). Subjects were randomly assigned to four groups according to the method of applying various forms of color information. The incoming color information was classified into four color conditions: yellow, red, green, blue. Each color was applied for 10 minutes for color adaptation. [Results] No significant difference was found for the tibialis anterior muscle, gastrocnemius muscle, and hamstring muscle, but there were significant differences in the quadriceps muscle between the four color conditions.[Conclusion] Input of various forms of color information will provide control of muscle in patients with an abnormal gait.

Key words: Balance, Color information, Gait
\end{abstract}

(This article was submitted Jun. 8, 2012, and was accepted Jul. 6, 2012)

\section{INTRODUCTION}

Keeping the body balanced is a dynamic activity that harmonizes both aspects of stability and mobility. It is also a combination of procedures that ensure a correct posture for the body, which includes reception and systematization of sensory inputs, motor programming, and execution ${ }^{1)}$. Maintaining body balance and walking are essential parts of daily life. Among the elements involved in the gait, the ability to control body balance depends on coordination of vestibular organs, proprioception, and the visual system. Sense of balance is the result of efforts to adapt to gravity, and stems from the function of vestibular organs. Vestibular organs are indispensable in perceiving and responding to actions and postures caused by changes in body position and space. If these factors fail to functional properly, body motions become slow, awkward, and more energyconsuming ${ }^{2}$.

Meanwhile, the visual system is a sensory system that depends on the function of retinal rods and cone cells. The former distinguish between light and shade, and the latter absorb photons through the visual pigments for color cognition $^{3)}$. In the visual system, the nerve conduction occurs to receive light stimulation, and the stimulation is transmitted to the visual center in the brainstem. It plays a primary role in maintaining a body orientation to place and balance. Also, as a means of spatial perception, the visual system mainly controls a body balance; it perceives distance or risk factors in the surrounding environment, provides information on the environment including the location and forms of movement, and provides data on the intensity and difficulty of the required movements for each part of the body ${ }^{4}$.

Many studies have reported that the visual system significantly affects control of a body balance through visual limitations and blocking ${ }^{5}$. People go through motor learning based on the visual feedback, and the visual stimulation affects a voluntary contraction of the lower limbs in the gait $^{6)}$. A previous study examined how wearing sunglasses for a prolonged period affects provision of black and white color information by the visual system, and reported that it decreased visual inputs and lowered balance ability as well as sense of distance and risk awareness, leading to a higher risk of a falling down ${ }^{4)}$.

These indicate that visual information has a great influence on body balance. The visual system receives different kinds of information from cone cells and retinal rods. Many studies have been undertaken on the relationship between the retinal rods and balance ability in light and shade, and yet, it is harder to find studies on the function of cone cells and reception of color information. Recently, a study examined the impact of wearing various colored glasses for a prolonged period, 
1164 J. Phys. Ther. Sci. Vol. 24, No. 11, 2012

Table 1. Lower limb muscle activation during the gait according to the various forms of color information

\begin{tabular}{lcrrrr}
\hline $\begin{array}{l}\text { Mus- } \\
\text { cle }\end{array}$ & No color & Red & Yellow & Green & Blue \\
\hline Qm* & $4538.62 \pm 1874.31$ & $1176.67 \pm 177.47$ & $1192.36 \pm 161.65$ & $1742.04 \pm 262.58$ & $2001.69 \pm 185.75$ \\
TA & $2111.82 \pm 213.33$ & $1920.09 \pm 231.44$ & $2258.64 \pm 263.11$ & $2375.31 \pm 441.78$ & $1878.41 \pm 232.71$ \\
Gm & $1146.14 \pm 106.98$ & $999.51 \pm 133.86$ & $1275.78 \pm 177.99$ & $1203.61 \pm 199.17$ & $1130.09 \pm 168.12$ \\
Hm & $2441.96 \pm 517.98$ & $1479.89 \pm 359.55$ & $1485.97 \pm 357.41$ & $2919.75 \pm 759.92$ & $1982.02 \pm 544.88$ \\
\hline
\end{tabular}

$(\mathrm{n}=20) \mathrm{p}<0.05, \operatorname{mean} \pm$ SE. RF: Rectus femoris muscle. TA: Tibialis anterior, GM: Gastrocnemius muscle. Hm: Hamstring muscle.

and reported that such an arrangement reduced visual input in people's daily living7). Another study examined how a gait and foot pressure are affected when colored glasses are suddenly removed after color adaptation, and it was shown that color information had a significant influence on the gait and foot pressure ${ }^{8}$. These studies provide objective and systematic information on the function of the visual system, and they underscore the influence of various colors on body balance and gait (ibid.). To date, not many studies have been undertaken to illuminate the relationship between muscle activation and color information in relation to gait. Given this circumstance, this study examined how various form of color information received by the cone cells lead to lower limb muscle activation, depending on the gait and balance control ability, and analyzed the impact of the subsequent visual adaptation on activities of daily living (ADL).

\section{SUBJECTS AND METHODS}

The experiment subjects included 20 healthy adults who received a full explanation of the objective and methods of the experiment and consented to participation. Among them, 15 were selected who had a corrected eyesight of 1.0 or higher and no eyesight-related issues - astigmatism, color blindness, color weakness, squint, or diplopia.

To examine the influence of various forms of color information on the lower limb muscle activation in the gait, the subjects were randomly asked to wear glasses tinted red, yellow, green, or blue (chroma 50, brightness 50). They were given ten minutes for adaptation and were asked to walk to measure muscle activation in the gait.

To measure the muscle activation in the gait, a surface EMG (Biopac Student Lab MP 36, Biopac System, Inc., Goleta, CA, USA) was used, and the received analog biosignals (physiological signals) were digitalized. Then the digitalized data were filtered and processed on a computer using the Acknowledge software. To eliminate measurement errors, prior to the experiment, alcohol was applied to the skin to cleanse the parts where the electrodes would be attached. A generous amount of electrolyte gel was applied to the electrodes, which were then attached to the skin and fixed with a paper tape. The locations of the electrodes - the rectus femoris, the tibialis anterior, the gastrocnemius, and the hamstring muscle - were decided based on other research and literature on electromyography. After measuring EMG signals of the muscles, their root mean square (RMS) values, which are approximate to actual outputs, were divided by the reference voluntary contraction (RVC) value and converted to $\% \mathrm{RVC}$ values.

\section{RESULTS}

Regarding the general characteristics of the 15 study subjects, their ages, heights, and weights were $25.2 \pm 3.21$, $169.68 \pm 6.6 \mathrm{~cm}$, and $63.937 \pm 12.019 \mathrm{~kg}$, respectively. The value of the muscle activation for the tibialis anterior was $2111.819 \pm 213.398$ with no color, 1920.094 \pm 231.441 for the color red, $2258.643 \pm 263.119$ for the color yellow, $2375.319 \pm 441.785$ for the color green, and $1878.411 \pm 232.71$ for the color blue. The tibialis anterior showed quite active muscle activation on average, but there was no significant difference statistically ( $\mathrm{p}>0.05)$.

The muscle activation of the gastrocnemius was $1146.1439 \pm 106.98274$ with no color, $999.5167 \pm 133.86908$ for the color red, $1275.7834 \pm 177.99223$ for the color yellow, $1203.6103 \pm 199.17820$ for the color green, and $1130.0977 \pm$ 168.12462 for color blue. The gastrocnemius also showed active muscle activation on average, but there was no significant difference statistically ( $\mathrm{p}>0.05$ ).

The muscle activation of the hamstring was $2441.9665 \pm 517.98090$ withno color, $1479.8977 \pm 359.55119$ for the color red, $1485.9712 \pm 357.41084$ for the color yellow, 2919.7580 \pm 759.92279 for the color green, and $1982.0290 \pm 544.88963$ for the color blue. All in all, the hamstring showed active muscle activation, but there was no significant difference statistically $(\mathrm{p}>0.05)$.

Meanwhile, muscle activation of the rectus femoris was $4538.685 \pm 1874.362$ with no color, $1176.673 \pm 177.474$ for the color red, $1192.364 \pm 161.655$ for the color yellow, $1742.041 \pm 262.584$ for the color green, and 2001.69 \pm 185.752 for the color blue, indicating high muscle activation on average as well as statistically significant differences $(\mathrm{p}<0.05)$ (Table 1).

\section{DISCUSSION}

This study examined the influence of various forms of color information on a person's gait and used a surface EMG system to measure the muscle activation of the rectus femoris, the tibialis anterior, the gastrocnemius, and the hamstring muscle to this effect. According to the experiment results, there was no statistically significant difference amongst input of different colors for the tibialis anterior, the gastrocnemius, and the hamstring muscle. In comparison, 
muscle activation of the rectus femoris was $4538.6 \pm 1874.3$ with no color, $1176.67 \pm 177.47$ for red color information, $1192.36 \pm 161.65$ for yellow color information, $1742.04 \pm$ 262.58 for green color information, and $2001.69 \pm 185.75$ for blue color information.

This suggests that, with colored glasses on, muscle activation of the rectus femoris declines in the gait as the visual system gradually adapts to the input of color information. The rectus femoris is related to primary extension of the knee joint and helps to control moving velocity of the center of mass in weight shift. In the gait, it slightly bends the knee joint, like a spring, in the heel strike stage in order to absorb the shock ${ }^{9}$. Thus, once the visual system adapts to the various forms of color information, muscle activation of the rectus femoris becomes lower, and the gait becomes more unstable as the rectus femoris engages less in weight shift, stabilization of the knee joint, and shock absorption.

When a person wears colored glasses for a prolonged time, it becomes difficult to receive correct information about the surroundings through the visual system. Without accurate information concerning the sense of position of each body part, it becomes hard to execute correct movements. In addition, a previous study showed that color information upsets the pattern of foot pressure changes in a normal gait ${ }^{8)}$. When exposed to color information, the lower limb muscles become less stable, and the distribution of the center of mass and weight shifting become less accurate in the gait as a result; this leads to a heightened risk of a falling down. Another study examined supply of color information through colored glasses for a prolonged period of time and reported that the amount of visual input decreased and failed to provide information required for stability control ${ }^{4)}$.

Based on these findings, it is surmised that wearing colored glasses for an extended period of time is likely to increase the risk of a falling down, especially for sick people or elderly people with a reduced balance ability. Also, it is likely to increase the risk of damage in sports activities such as baseball, football, basketball, and running, which involve unstable and unexpected body movements compared with a normal gait. For a broader clinical application of the information on color adaptation, future studies should examine a wider range of colors and their impact on activation of various muscles.

\section{ACKNOWLEDGEMENTS}

This research was supported by a Kyungsung University Research Grant in 2012.

\section{REFERENCES}

1) Eng JJ, Chu KS: Reliability and comparison of weigh-bearing ability during standing tasks for individuals with chronic stroke. Arch Phys Med Rehabil, 2002, 83: 1138-1144. [Medline] [CrossRef]

2) Guyton AC: Function of the human body. Sunders Co, 1959, 72: 305-357.

3) Swanson WH, Cohen JM: Color vision. Ophthalmol Clin North Am, 2003, ***: 179-203. [Medline] [CrossRef]

4) Taylor LP: Taylor's Manual of Physical Evaluation and Treatment vol II. US. SLACK. 1990.

5) Diener HC, Horak FB, Nashner LM: Influence of stimulus parameter on human postural responses. J Neurophysiol, 1988, 59: 1888-1905. [Medline]

6) Lackner JR, Dizo P: Visual stimulation affects the perception of voluntary leg moverments during walking. Perception, 1988, 17: 71-80. [Medline] [CrossRef]

7) $\mathrm{Ha} \mathrm{KJ}$ : The effect of various color information on gait and balance of normal. Kyoungsung University Master's thesis. 2010.

8) Lee SY, Choi SH, Lee SK: Effects of Adaptation and Sudden Remove of Various Color Information on Plantar Foot Pressure during Gait in Normal Adults. J Korean Soc Phys Med, 2011, 6: 391-396.

9) Donald A.: Neumann, Kinesiology of the Muscu- loskeletalSystem (2nd ed). Singapore: Mosby, 2002. 